\title{
Genetic instability associated with insertion of IS6100 into one end of the Streptomyces lividans chromosome
}

\author{
Gülsüm Güneș, † Barry Smithł and Paul Dyson
}

Author for correspondence: Paul Dyson. Tel: +44 1792 295667. Fax: +44 1792295447.

e-mail:p.j.dyson@swansea.ac.uk

Molecular Biology Research Group, School of Biological Sciences, University of Wales Swansea, Swansea SA2 8PP, UK

\begin{abstract}
Analysis of 548 recombinant strains of Streptomyces lividans carrying chromosomal insertions of IS6100 revealed that six mutants contained DNA amplifications. The amplifications differed in size but included IS6100 sequences. Hybridization with representative cosmid clones containing sequences from the unstable regions of the chromosome indicated that, in each mutant, DNA rearrangements affected just one of the chromosome ends. The amplifications were derived either from a region immediately proximal to the terminal inverted repeat (TIR) or further distal, from a previously characterized type I amplifiable unit of DNA. There was no evidence for extensive deletions accompanying the amplifications and chromosome linearity was maintained with, at least in five mutants, clear evidence for no loss of either TIR. The nature of the rearrangements provides evidence that insertions affecting the integrity of a chromosome end can contribute to genetic instability in Streptomyces.
\end{abstract}

Keywords: Streptomyces lividans, insertion sequence, IS6100, genetic instability

\section{INTRODUCTION}

The linear Streptomyces chromosome can exhibit highfrequency genetic instability, undergoing spontaneous deletions at rates that can be higher than $0 \cdot 1 \%$ of spores (reviewed by Volff \& Altenbuchner, 1998). These deletions often affect the chromosome ends, including the terminal inverted repeats (TIRs), the $5^{\prime}$ ends of which are covalently bound by a terminal protein believed to be essential for replication of terminal sequences (Lin et al., 1993). In the absence of this complex, viability can be maintained by chromosomal circularization. Moreover, tandem amplification of specific chromosomal sequences is frequently associated with the deletions.

These phenomena have been investigated in some detail in Streptomyces lividans. In this species, the extremities of the chromosome consist of $30 \mathrm{~kb}$ TIRs, which contain

\footnotetext{
† Present address: Gebze Institute for Advanced Technology, 41400 Gebze, Kocaeli, Turkey.

‡Present address: Marie Curie Research Institute, The Chart, Oxted RH8 OTL, UK.

Abbreviations: AUD, amplifiable unit of DNA; TIR, terminal inverted repeat.
}

two transposable elements: Tn4811 and IS1372 (Lin et al., 1993; Fischer et al., 1996). A physical genetic map of a $1 \mathrm{Mb}$ region spanning both chromosome ends has been assembled (Redenbach et al., 1993). The regions immediately adjacent to the TIRs lack any housekeeping genes; indeed, in the related Streptomyces coelicolor A3(2), with the exception of $\arg G$, no essential genes (defined as genes needed for growth in laboratory conditions) are located within $1.2 \mathrm{Mb}$ of either end (Redenbach et al., 1996). Sequential deletions in one chromosomal arm can remove a TIR, a chloramphenicol-resistance marker and $\arg G$. The majority of these mutants contain a highly amplified $5.7 \mathrm{~kb}$ sequence which defines, or is close to, the deletion end point (Altenbuchner \& Cullum, 1984; Betzler et al., 1987). This sequence originates from a type II amplifiable unit of DNA $\left(\mathrm{AUD}_{5.7}\right)$ in progenitor strains; type II AUDs give rise to amplified sequences of identical structure in independently isolated mutants and consist of an internal sequence flanked by direct repeats of at least $0 \cdot 8 \mathrm{~kb}$ (Volff \& Altenbuchner, 1998). Instability affecting the other chromosomal arm has been shown to cause large deletions, including the TIR, often ending at amplified sequences of different sizes in independently isolated mutants (Rauland et al., 1995). These arise from type I AUDs, which are characteristically heterogeneous 
in their size and end points, but are derived from overlapping chromosomal regions.

The evolution of this genetic organization, with the absence of essential genes in unstable regions and AUD structures that can amplify, possibly to protect against further deletion, implies that genetic instability may not be an unusual fate for a streptomycete, even when living in its natural environment. This instability may be associated with chromosomal linearity, because circularized derivatives lack functions to ensure their stable propagation. Models to account for frequent formation of terminal deletions propose that collapsed replication forks may be repaired by homologous recombination between chromosomes in the multinucleoid mycelium (Volff \& Altenbuchner, 1998). The frequency of these events is likely to be stimulated by genotoxic agents which are well known to increase levels of spontaneous instability. Alternatively, replicative transposition of a transposable element normally resident in an unstable region could promote deletion formation (Chen, 1996; Volff \& Altenbuchner, 1998). Although there is no evidence to date for transposition of an indigenous element causing rearrangements of unstable regions, in this study we characterize genetic rearrangements associated with transposition of the heterologous IS6100 into one chromosome end of S. lividans. IS6100, originating from Mycobacterium fortuitum, belongs to the IS6 family of transposable elements and generates cointegrates as the end product of its replicative transposition mechanism (Martin et al., 1990; Smith \& Dyson, 1995).

\section{METHODS}

Growth conditions, strains and transposon mutagenesis. IS6100 was introduced into S. lividans strain 66 (Hopwood et al., 1985) on the temperature-sensitive delivery vector pUCS30 (Smith \& Dyson, 1995) by PEG-mediated transformation of protoplasts and selection on regeneration media as described previously (Smith \& Dyson, 1995). Individual insertion mutants were obtained from outgrowing sectors of colonies grown at the non-permissive temperature for replication of pUCS30, as has been described previously (Smith \& Dyson, 1995), in the absence of thiostrepton-induction of transposition. Purified transposition mutants were used to inoculate a tryptic-soy-containing broth (Dyson \& Schrempf, 1987), and grown, without antibiotic selection, at $40{ }^{\circ} \mathrm{C}$ prior to DNA isolation. Antibiotic resistance of transposition mutants was tested on GHM medium (containing $\mathrm{l}^{-1}: 4 \mathrm{~g}$ glucose, $4 \mathrm{~g}$ yeast extract, $10 \mathrm{~g}$ malt extract, $2 \mathrm{~g} \mathrm{CaCO}_{3}, 12 \mathrm{~g}$ agar) supplemented with either chloramphenicol $\left(8 \mu \mathrm{g} \mathrm{ml}^{-1}\right)$ or tetracycline $\left(25 \mu \mathrm{g} \mathrm{ml}^{-1}\right)$. Prototrophy was tested for by growth on minimal medium (Hopwood et al., 1985). Escherichia coli strain $\mathrm{F}^{-} \mathrm{Z}^{-} \Delta \mathrm{M} 15$ (Rüther et al., 1981) was employed as a host to maintain the other plasmids and cosmids used in this study. These plasmids were introduced by a standard calcium-chloride-mediated transformation procedure (Sambrook et al., 1989). The plasmids and cosmids used as hybridization probes were: pUCS19 (Smith \& Dyson, 1995), containing IS6100; pJD201 (Betzler et al., 1987), containing an $\mathrm{AUD}_{5 \cdot 7}$ repeat sequence; pSL100 (Betzler et al., 1987), containing $\arg G$; and cosmids containing either $S$. lividans telomeric sequences: 68/7, 11/9, 26, 66 and A7
(Redenbach et al., 1993) or S. coelicolor A3(2) DNA: F37 and F42 (Redenbach et al., 1996).

DNA manipulations. S. lividans chromosomal DNA was isolated as described previously (Dyson \& Schrempf, 1987). Plasmid and cosmid DNA was isolated from E. coli by alkaline lysis (Sambrook et al., 1989). Restriction enzymes were from either Life Technologies or New England Biolabs, and were used as recommended by the suppliers. Restriction fragments required for probe synthesis were purified by electrophoresis in $0.8 \%$ agarose gels prepared in TAE buffer (Tris/ acetate/EDTA; Sambrook et al., 1989) and subsequently isolated from sodium-iodide-dissolved gel slices by binding to glassmilk using a Geneclean II kit, following the manufacturer's instructions (Bio 101).

Restricted chromosomal DNA was electrophoresed in $0.8 \%$ agarose gels run in HEPES buffer (Ray et al., 1992) to avoid Tris-mediated DNA cleavage. The extent of amplification was estimated by densitometric analysis of restricted amplified DNA fragments in each gel lane after ethidium bromide staining of the gel. A Quantity One (Bio-Rad) software package was used for the analysis. The sizes of individual amplified restriction fragments were calculated by comparison with a $\lambda$ HindIII size marker. The DNA was transferred to a Hybond-N nylon membrane (Amersham) using a vacuumblotting system (Stratagene). An IS6100-specific probe was prepared by gel purification of a $0.9 \mathrm{~kb} S m a \mathrm{I}$ fragment of pUCS19. An internal pUCS30 probe was prepared by gel purification of a $2.3 \mathrm{~kb}$ HindIII-EcoRI pUCS30 fragment that spans a unique XhoI site. Probes were labelled with digoxigenin-11-dUTP by random priming using a kit from Boehringer Mannheim. Hybridization conditions and subsequent detection by colour reaction with 4-nitroblue tetrazolium chloride and 5-bromo-4-chloro-3-indolyl phosphate were according to the manufacturer's instructions (Boehringer Mannheim).

Inserts for PFGE were prepared from 4-d-old cultures grown in tryptic soy medium, as described previously (Leblond et al., 1993). Undigested or AflII-digested DNA was separated by PFGE in $1 \%$ agarose gels in a contour-clamped homogeneous electric field system (Chu et al., 1986) supplied by Bio-Rad. The running buffer was $0.5 \times$ TBE (Sambrook et al., 1989) supplemented with $50 \mu \mathrm{M}$ thiourea. Pulse times were optimized with respect to the length of DNA to be separated.

\section{RESULTS AND DISCUSSION}

\section{Isolation and characterization of IS6100 transposon mutants containing amplified DNA sequences}

Analysis of DNA isolated from 548 individual transposon mutants showed that six clones (i.e. $1 \%$ ) contained amplified DNA fragments, and although some of these six shared amplified BamHI restriction fragments of similar molecular mass, no two possessed identical profiles of amplified fragments. Southern hybridization with an IS6100 probe revealed that in each case at least one of the amplified fragments contained the insertion sequence (Fig. 1). Amplification of intervening sequences between two directly repeated copies of an insertion sequence has been observed in other bacteria (Peterson \& Rownd, 1985). If this had occurred here, concatameric amplification of the cointegrate of pUCS30 would have resulted in a single amplified $\mathrm{Bam} \mathrm{HI}$ restriction fragment of $15 \cdot 4 \mathrm{~kb}$. However, assuming the amplified restriction 
(a)

(b)

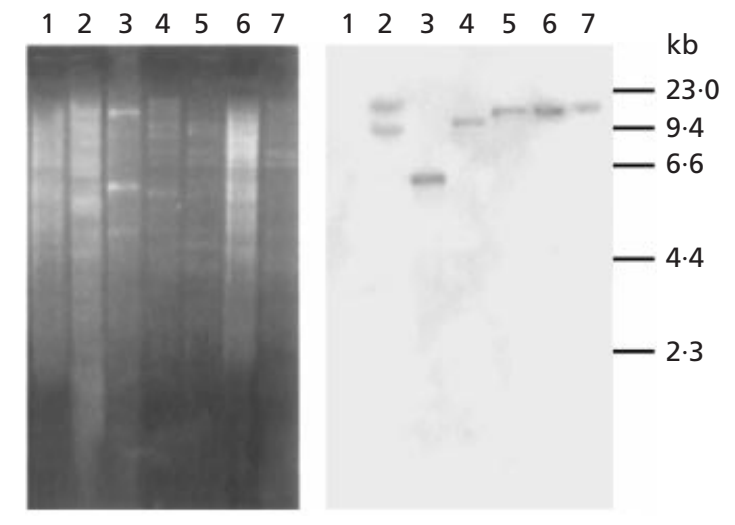

Fig. 1. Amplification of IS6100 sequences in transposon mutants of S. lividans. BamHI-digested genomic DNA from wild-type, and mutants 2E, 3N, 19C, 18l, 9A and 5B (lanes 1-7, respectively) was separated by gel electrophoresis (a). A Southern blot of this DNA was hybridized with an 156100 probe (b). The positions of size markers ( $\lambda$ HindIII) are indicated.

Table 1. Characteristics of amplification mutants

\begin{tabular}{|lccc|}
\hline \multirow{2}{*}{ Mutant } & \multicolumn{2}{c|}{ Amplified DNA } & $\begin{array}{c}\text { Colony } \\
\text { phenotype* }\end{array}$ \\
\cline { 2 - 3 } & Size $(\mathbf{k b})$ & Copy no. & \\
\hline 2E & 85 & 4 & Amy $^{-}$Pig $^{-}$ \\
3N & 65 & 15 & Amy $^{+}$Pig $^{+}$ \\
5B & 55 & 7 & Amy $^{-}$Pig $^{-}$ \\
9A & 80 & 5 & Amy $^{+}$Pig $^{+}$ \\
18I & 270 & 5 & Amy $^{+}$Pig $^{+}$ \\
19C & 280 & 5 & Amy $^{+}$Pig $^{+}$ \\
\hline
\end{tabular}

* Amy, aerial mycelial formation; Pig, production of pigmented antibiotics.

fragments in each mutant were derived from single DNA amplifications, the total sizes of the amplified units were estimated to vary from 55 to $280 \mathrm{~kb}$ in the different mutants (Table 1). A $15.4 \mathrm{~kb}$ fragment comigrating with linearized pUCS30 and hybridizing with the IS6100 probe could not be detected in any of the six strains (results not shown). The extent of amplification was estimated to vary between 4 - and 15 -fold in the different mutants (Table 1).

Hybridization with a probe specific for non-IS6100 sequences of pUCS30 also indicated unusual arrangements of the integrated sequences in these amplifications. Hybridization of this probe to BamHI-digested chromosomal DNA from non-amplified transposon mutants carrying a 'normal' cointegrate reveals a single band varying in size in different isolates. However, this probe hybridized to three amplified restriction fragments of strain 9A, two amplified restriction fragments from mutants 19C, 3E and 18I, one amplified fragment from mutant $3 \mathrm{~N}$ (the hybridizing fragments from each mutant were of different molecular mass), and not at all to DNA from strain $5 \mathrm{~B}$.

Examination of the morphological and pigmentation phenotypes of the mutants when grown on complete medium with glucose as the carbon source revealed that four mutants sporulated normally and exhibited normal pigmentation, whereas two mutants were deficient in both aerial mycelium formation and pigmentation (Table 1). We also examined the phenotypes with regard to typical unstable traits in S. lividans. All six mutants were resistant to chloramphenicol and tetracycline, and were prototrophic. Southern hybridization with AUD $_{5 \cdot 7}$ and $\arg G$ probes against BamHI-restricted DNA from the wild-type and the six mutants did not detect any rearrangements associated with this chromosomal end (data not shown). The absence of rearrangements was confirmed by hybridizing a cosmid $11 / 9$ probe, containing approximately $12 \mathrm{~kb}$ of TIR sequence (see Fig. 2), which detected a similar $900 \mathrm{~kb}$ AflII PFGE fragment from this chromosomal end in wild-type and in all six mutants (data not shown).

\section{Rearrangements affect one chromosome end}

The extent of the rearrangements in each mutant was analysed by hybridizing cosmid probes encompassing the other chromosome end against BamHI-digested wild-type and mutant DNA separated by conventional electrophoresis and AflII-digested DNA separated by PFGE. These analyses showed that there were no extensive deletions in any of the mutants, and that the amplifications were derived from either of two regions (Fig. 2).

The end point of one amplifiable region was proximal to the innermost end of the TIR. Mutants $2 \mathrm{E}, 3 \mathrm{~N}, 5 \mathrm{~B}$ and 9A contained different-sized overlapping amplified sequences from this region, all detected by hybridization with cosmid 26. The rearrangements in strain $2 \mathrm{E}$ were confirmed by PFGE and hybridization with the cosmid 66 probe, indicating an absence of both the 22 and $300 \mathrm{~kb}$ AflII fragments, and the presence of a novel fragment of approximately $680 \mathrm{~kb}$. The other cosmid probes detected similar fragments in both wild-type and $2 \mathrm{E}$, including the $80 \mathrm{~kb}$ AflII fragment proximal to the TIR. In contrast, PFGE analysis of mutant $3 \mathrm{~N}$ revealed that the rearrangements extended into the TIR-proximal $80 \mathrm{~kb}$ AflII fragment, which was absent, as were the $22 \mathrm{~kb}$ and $300 \mathrm{~kb}$ AflII fragments; these were replaced with fragments of approximately $510 \mathrm{~kb}$ and $>1 \cdot 2 \mathrm{Mb}$. Hybridization with cosmid probe 66 against BamHIdigested DNA revealed that the majority of wild-type fragments were retained in mutant $3 \mathrm{~N}$, indicating the limited extent of any rearrangements flanking the amplified DNA. This was confirmed by hybridization with the other cosmid probes, thus providing no evidence for other rearrangements. The TIR-proximal $80 \mathrm{~kb}$ AflII fragment of mutant $5 \mathrm{~B}$ was found to be retained, but rearrangements affected the $22 \mathrm{~kb}$ and $300 \mathrm{~kb}$ AflII fragments, which were replaced by a $450 \mathrm{~kb}$ 
(a) $100 \mathrm{~kb}$

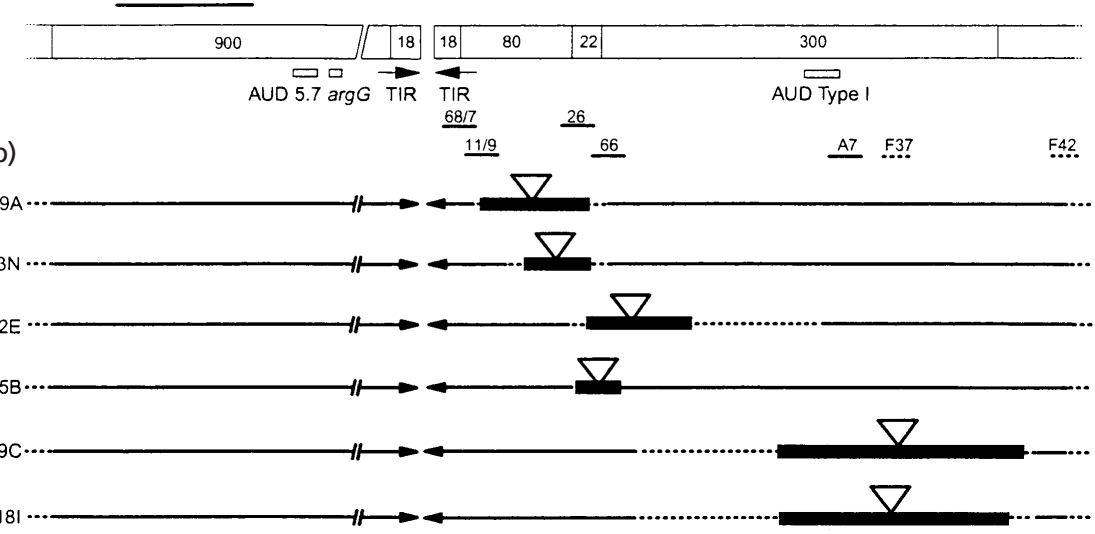

Fig. 2. Extent of rearrangements in amplified mutants. (a) A map of the wild-type chromosome ends indicating the sizes (kb) of the AfIII fragments, and the positions of the AUD 5.7 , AUD type I, argG and the TIRs (from Redenbach et al., 1993). The locations of the cloned S. lividans sequences present in the cosmids used as hybridization probes are indicated by solid bars under the AfIII restriction map; the approximate equivalent locations of the S. coelicolor DNA in cosmids F37 and F42 are represented by dashed bars. (b) Approximate positions of the amplified DNA in each mutant are represented by solid boxes, including IS6100 sequences (triangles) whose precise locations in the various amplified structures were not determined. Dashed lines flanking the amplified DNA represent the furthest possible extent of any adjacent rearrangements.

(a)

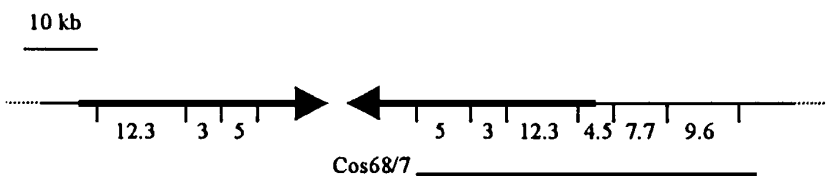

(b)

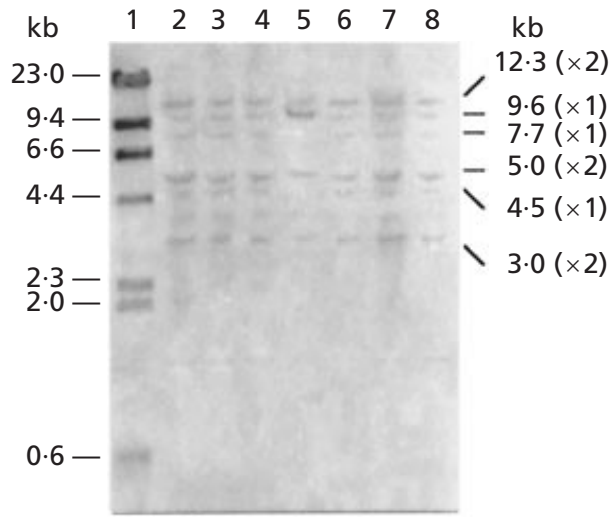

Fig. 3. Retention of the TIR proximal to the amplifications. (a) A Xhol restriction map of the TIRs and the immediate flanking region contained in cosmid 68/7, the extent of which is denoted beneath the map. The sizes (kb) of $X$ hol restriction fragments are indicated (from Redenbach et al., 1993; Volff \& Altenbuchner, 1997). (b) Hybridization with a cosmid 68/7 probe against Xhol-digested chromosomal DNA from wild-type and mutants 2E, 3N, 9A, 5B, 19C and 18I (lanes 2-8, respectively). The sizes $(\mathrm{kb})$ of the TIR-specific fragments detected and their copy number are indicated to the right of the blot. An amplified $9.6 \mathrm{~kb}$ Xhol fragment is evident in mutant $9 \mathrm{~A}$. A $\lambda$ Hindlll size marker is in lane 1.

fragment. Hybridization with cosmid probe 66 against BamHI-digested 5B DNA revealed a novel $19 \mathrm{~kb}$ amplified DNA fragment, a novel single-copy $12 \mathrm{~kb}$ fragment and two unrearranged single-copy fragments, indicating the limit to the extent of rearrangements in this mutant. In the overlapping amplifications contained in these four mutants, the end point of the $80 \mathrm{~kb}$ amplification of mutant 9A mapped closest to the TIR, since it was detected by probes of both cosmids $11 / 9$ and $68 / 7$ (Fig. 3). This amplification overlaps the junction between the TIR and flanking unique sequence DNA, which, from sequence analysis, contains numerous long direct repeats (Volff \& Altenbuchner, 1997). The other amplification end point was concluded to lie in the region spanned by cosmid 66, which detected novel amplified and single-copy fragments of $25 \mathrm{~kb}$ and $13 \mathrm{~kb}$, respectively, in addition to several wild-type single-copy fragments. This was confirmed by the loss of the 80,22 and $300 \mathrm{~kb}$ AflII fragments with the appearance of at least one novel AflII fragment of $410 \mathrm{~kb}$. None of the four TIR proximal amplifications contained an internal AflII site, despite originating from a chromosomal region represented by cosmids 26 and 66, in which AflII sites are present. This is evidence for internal deletions 
associated with the rearrangements in these amplified mutants.

Strains 19C and 18I contained larger amplifications, of 280 and $270 \mathrm{~kb}$, respectively. These overlapped a type I AUD region more distal to the TIR. The presence of this AUD had previously been described in association with rearrangements in 2-deoxygalactose-resistant mutants of S. lividans strain U001 (Rauland et al., 1995). The amplifications in mutants $19 \mathrm{C}$ and $18 \mathrm{I}$ were both detected with cosmid probe A7 containing these AUD type I sequences (Fig. 2); hybridization against AflII digests showed that the $300 \mathrm{~kb}$ fragment of wild-type was absent, whereas $280 \mathrm{~kb}$ and $270 \mathrm{~kb}$ unit length amplified AflII fragments were present in strains 19C and 18I, respectively. To investigate further the extent of the rearrangements in these two mutants, two cosmids from the $S$. coelicolor genome library were used as probes: cosmid F37, containing sequences adjacent but TIR-distal to a homologous AUD region, and cosmid $\mathrm{F} 42$, containing a region some $120 \mathrm{~kb}$ further inside the chromosome (Redenbach et al., 1996). Whereas the cosmid F37 probe hybridized to amplified DNA fragments in both mutants, including a common $23 \mathrm{~kb}$ BamHI fragment of mutant 18I identified by the cosmid A7 probe, the hybridization patterns of the mutants revealed with the F42 probe were similar to the wildtype. Moreover, for both mutants, the other S. lividans TIR proximal cosmid probes detected similar hybridizing patterns to the wild-type, suggesting retention of the chromosome end. Mutants 19C and 18I therefore differ from most 2-deoxygalactose mutants derived from strain U001, in which amplifications define end points of large deletions that remove the chromosome end (Rauland et al., 1995). Interestingly, the formation of these particular rearrangements required the prior integration of plasmid pMT664, carrying a copy of IS466, into the chromosome of the progenitor U001. However, as the site of integration was $1.75 \mathrm{Mb}$ distal from the TIR, and sequences homologous to pMT664 were not found associated with the rearrangements, the role of the integrated plasmid and insertion sequence in provoking the instability is unclear.

\section{Linear topology of the mutant chromosomes}

The retention of at least one unrearranged chromosome end suggested that the mutant chromosomes would be linear. This was confirmed by comparing the migration of the chromosome in PFGE samples prepared with or without proteinase $\mathrm{K}$ treatment. Chromosomal or linear plasmid DNA with bound terminal proteins does not enter a gel during PFGE, whereas the DNA in samples in which these proteins are digested migrates according to its molecular mass (Lin et al., 1993). This analysis revealed that, just as in the wild-type, the mutant chromosomes were linear and, moreover, each mutant strain retained the $50 \mathrm{~kb}$ linear plasmid SLP2 (data not shown). Most $S$. lividans mutants containing amplified DNA have been found to contain circularized chromosomes (Volff \& Altenbuchner, 1998). Where chromosomal linearity was maintained only one TIR was retained. Due to the duplication of TIR sequences, the question of whether both TIRs are present is complicated. To investigate this further in the IS6100amplified mutants, we looked for the presence of a unique $4.5 \mathrm{~kb}$ XhoI fragment which overlaps the innermost portion of the TIR in question (Redenbach et al., 1993; Volff \& Altenbuchner, 1997). Xhol chromosomal digests were probed with cosmid 68/7, which contains a region overlapping the TIR together with adjacent unique sequences. This revealed the $4.5 \mathrm{~kb}$ fragment in strains $2 \mathrm{E}, 3 \mathrm{~N}, 5 \mathrm{~B}, 18 \mathrm{I}$ and 19C (Fig. 3). Both this fragment, and the adjacent $7.7 \mathrm{~kb}$ fragment from the unique region, were not evident in strain 9A, confirming rearrangements associated with amplification of the TIR flanking sequence. The remaining TIR-internal XhoI fragments could be attributed to the TIR of the unrearranged chromosome end. Although the strength of the hybridization signals was consistent with these fragments being duplicated, and hence both TIRs being present, this requires confirmation.

\section{Concluding remarks}

Transposition of IS6100 into one end of the S. lividans chromosome stimulated rearrangements in this region, resulting in low-level amplification of the insertion sequence and adjacent chromosomal DNA. In contrast to commonly described rearrangements affecting the $S$. lividans chromosome ends, the amplifications were not accompanied by extensive deletions affecting terminal sequences and chromosomal linearity was maintained. Linearity was also maintained in some $S$. ambofaciens mutants carrying deletions affecting one chromosome end (Fischer et al., 1997). The expected cointegrate structures resulting from simple intermolecular transposition of IS6100 were not present, indicating that in the derivation of the mutants additional recombination events, leading to amplification and affecting both the inserted DNA and sequences internal to the TIR, are likely to have occurred. The results suggest that insertions affecting the integrity of a chromosomal end can provoke recombination, and indicate that transposition can contribute to the high-frequency genetic instability of streptomycetes. Due to the lack of essential genes at the ends, the region may act as a 'sink' for the accumulation of transposable elements, and these could subsequently act to destabilize the chromosome.

\section{ACKNOWLEDGEMENTS}

G.G. was supported by the Gebze Institute for Advanced Technology, Turkey; B.S. was supported by a BBSRC studentship. We are grateful to M. Redenbach and J. Cullum for the cosmids used in the study. Special thanks go to Professor Sir David Hopwood FRS for the inspiration and encouragement he has provided over several years.

\section{REFERENCES}

Altenbuchner, J. \& Cullum, J. (1984). DNA amplification and an unstable arginine gene in Streptomyces lividans 66. Mol Gen Genet 195, 134-138. 
Betzler, M., Dyson, P. \& Schrempf, H. (1987). Relationship of an unstable $\arg G$ gene to a 5.7-kilobase amplifiable DNA sequence in Streptomyces lividans 66. J Bacteriol 169, 4804-4810.

Chen, C. W. (1996). Complications and implications of linear bacterial chromosomes. Trends Genet 12, 192-196.

Chu, G., Vollrath, D. \& Davis, R. W. (1986). Separation of large DNA molecules by contour-clamped homogeneous electric fields. Science 234, 1582-1585.

Dyson, P. \& Schrempf, H. (1987). Genetic instability and DNA amplification in Streptomyces lividans 66. J Bacteriol 169, 4796-4803.

Fischer, G., Decaris, B. \& Leblond, P. (1997). Occurrence of deletions, associated with genetic instability in Streptomyces ambofaciens, is independent of the linearity of the chromosomal DNA. J Bacteriol 179, 4553-4558.

Fischer, J., Maier, H., Viell, P. \& Altenbuchner, J. (1996). The use of an improved transposon mutagenesis system for DNA sequencing leads to the characterization of a new insertion sequence of Streptomyces lividans 66. Gene 180, 81-89.

Hopwood, D. A., Bibb, M. J., Chater, K. F. \& 7 other authors (1985). Genetic Manipulation of Streptomyces: a Laboratory Manual. Norwich: John Innes Foundation.

Leblond, P., Redenbach, M. \& Cullum, J. (1993). Physical map of the Streptomyces lividans 66 genome and comparison with that of the related strain Streptomyces coelicolor A3(2). J Bacteriol 175, 3422-3429.

Lin, Y. S., Kieser, H., Hopwood, D. A. \& Chen, C. (1993). The chromosomal DNA of Streptomyces lividans 66 is linear. Mol Microbiol 10, 923-933.

Martin, C., Timm, J., Rauzier, J., Gomez-Lus, R., Davies, J. \& Gicquel, B. (1990). Transposition of an antibiotic resistance element in mycobacteria. Nature 345, 739-743.

Peterson, B. C. \& Rownd, R. H. (1985). Drug resistance gene amplification of plasmid NR1 derivatives with various amounts of resistance determinant DNA. J Bacteriol 161, 1042-1048.
Rauland, U., Glocker, I., Redenbach, M. \& Cullum, J. (1995). DNA amplifications and deletions in Streptomyces lividans 66 and the loss of one end of the linear chromosome. Mol Gen Genet 246, 37-44.

Ray, T., Weaden, J. \& Dyson, P. (1992). Tris-dependent sitespecific cleavage of Streptomyces lividans DNA. FEMS Microbiol Lett 96, 247-252.

Redenbach, M., Flett, F., Piendl, W., Glocker, I., Rauland, U., Wafzig, O., Kliem, R., Leblond, P. \& Cullum, J. (1993). The Streptomyces lividans 66 chromosome contains a $1 \mathrm{MB}$ deletogenic region flanked by two amplifiable regions. Mol Gen Genet 241, 255-262.

Redenbach, M., Kieser, H. M., Denapaite, D., Eichner, A., Cullum, J., Kinashi, H. \& Hopwood, D. A. (1996). A set of ordered cosmids and a detailed genetic and physical map for the $8 \mathrm{Mb}$ Streptomyces coelicolor A3(2) chromosome. Mol Microbiol 21, 77-96.

Rüther, U., Koenen, M., Otto, K. \& Müller-Hill, B. (1981). pUR222, a vector for cloning and rapid chemical sequencing of DNA. Nucleic Acids Res 9, 4087-4098.

Sambrook, J., Fritsch, E. F. \& Maniatis, T. (1989). Molecular Cloning: a Laboratory Manual, 2nd edn. Cold Spring Harbor, NY : Cold Spring Harbor Laboratory.

Smith, B. \& Dyson, P. (1995). Inducible transposition in Streptomyces lividans of insertion sequence IS6100 from Mycobacterium fortuitum. Mol Microbiol 18, 933-941.

Volff, J.-N. \& Altenbuchner, J. (1997). Nucleotide sequence analysis of the junctions of the terminal inverted repeats of the Streptomyces lividans linear chromosome. Mol Gen Genet 253, 761-765.

Volff, J.-N. \& Altenbuchner, J. (1998). Genetic instability of the Streptomyces chromosome. Mol Microbiol 27, 239-246.

Received 25 February 1999; revised 17 May 1999; accepted 27 May 1999. 